\title{
Knowledge, Attitudes, and Practices Study on Hand Hygiene Among Imam Hossein Hospital's Residents in 2013
}

\author{
Mahmoud Nabavi ${ }^{1}$; Mostafa Alavi-Moghaddam ${ }^{2}$; Latif Gachkar ${ }^{3}$; Mohammad Moeinian ${ }^{1, *}$ \\ ${ }_{1}^{1}$ Department of Infectious Diseases and Tropical Medicine, Imam Hossein Hospital, Shahid Beheshti University of Medical Sciences, Tehran, IR Iran \\ ${ }_{3}^{2}$ Department of Emergency, Imam Hossein Hospital, Shahid Beheshti University of Medical Sciences, Tehran, IR Iran \\ 3 Infectious Diseases and Tropical Medicine Research Center, Shahid Beheshti University of Medical Sciences, Tehran, IR Iran \\ *Corresponding Author: Mohammad Moeinian, Department of Infectious Diseases and Tropical Medicine, Imam Hossein Hospital, Shahid Beheshti University of Medical Sciences, \\ Tehran, IR Iran. Tel:+98-2177543634, Fax:+98-2177543634, E-mail: moeinianmohammad@yahoo.com
}

Received: April 26, 2014; Revised: May 29, 2014; Accepted: July 3, 2014

\begin{abstract}
Background: Hand hygiene is considered one of the most important infection control measures for preventing health care-associated infections. Although the techniques involved in hand hygiene are simple, compliance with hand hygiene recommendations is poor worldwide.

Objectives: We sought to perform a knowledge, attitudes, and practices (KAP) study on hand hygiene among medical residents at Imam Hossein hospital, Tehran, Iran.

Patients and Methods: This cross-sectional KAP study was conducted among medical residents in Imam Hossein hospital, Iran, 2013. All medical residents from different wards were invited to participate in this study (270 in total). The world health organization questionnaires and an observational checklist were used to collect data. The $\chi^{2}$ test and the Fisher exact test were utilized to analyze the qualitative variables. Since the quantitative variables had no normal distribution, the Mann-Whitney test and the Kruskal-Wallis method were employed. A P value < 0.05 was considered statistically significant. The data were analyzed using SPSS, version 17.

Results: The mean overall score of the residents' knowledge was 14.2 \pm 2.6 (mean \pm SD). The residents received weak scores in attitudes and practices. Forty-nine percent $(n=124)$ of the residents responded to the questions on attitudes toward hand hygiene, and only $20.16 \%(n=$ 25) managed to identify the correct answer. Moreover, $3.1 \%(n=8)$ of the residents adhered to the 8 standard steps, $12.1 \%(n=31)$ washed their hands for 20 - 30 seconds, and only 2 residents observed the sequences of hand hygiene. Additionally, none of the residents performed hand washing with available means (water and hand-washing liquid) in the morning visit hours.

Conclusions: Concerning hand hygiene, the residents had moderate knowledge but overall poor attitudes and practices. The present study underscores the need for further improvement in the existing training programs to address the gaps in KAP regarding hand hygiene.
\end{abstract}

Keywords: Hand Hygiene; Medical Residents; KAP Study; Iran

\section{Background}

Health care-associated infections are a serious problem in health care services as they may cause prolonged hospital stays, high mortality, long-term disability, and excess health care costs. Most health care-associated infections can be transmitted from patient to patient via the hands of health care workers. In other words, health care workers' hands due to poor hand hygiene are the most usual type of vehicle for the transmission of health careassociated infections (1).

Effective hand hygiene is the simplest proven method to reduce the prevalence of health care-associated infections. Unfortunately, the prevalence of these infections continues to rise, and it is estimated that annually about hundreds of millions of patients suffer from health care-associated infections the world over. Therefore, infection control is necessary to reduce the high levels of health care-associated infections, and the importance of hand hygiene in the control of infection cannot be overemphasized $(1,2)$.
Hand hygiene is a general term referring to any action of hand cleansing by using water and detergent and/or the use of alcohol-based hand sanitizers for the removal of transient microorganisms from hands (3). Although many communities have guidelines on hand hygiene for health care settings, many researchers have found that measuring adherence to the hand hygiene guidelines is not a simple task. Experts in quality improvement have suggested that a multidisciplinary strategy is necessary to improve hand hygiene, including protocols, training, engineering controls and equipment, and routine observation and feedback (4).

Most would agree that hand hygiene is of critical importance, but numerous studies have shown that adherence to hand hygiene recommendations remains low and that improvement efforts frequently lack sustainability $(5,6)$. This is may be due to several constraints such as heavy work load, high number of clinical procedures, and skin 
conditions of health care workers (7).

In Asia, there is a paucity of studies (8-11) exploring this subject, although the prevalence of health care-associated infections is high in this region. Most of these studies have explored the knowledge, attitudes, and practices (KAP) of doctors and nurses with only a few including medical residents $(8,9)$. Infection control and aseptic techniques are taught to many Iranian medical residents by tutors during rotation exposures. We believe that it is essential that the effectiveness of the training programs of residents in this regard be fully explored. This would be useful in identifying gaps in KAP apropos hand hygiene with a view to improving the existing training programs and promoting good practices and work ethics in the future. However, our literature review failed to yield any study evaluating the level of knowledge, attitude, and behavior change following the routine training among Iranian medical residents.

There is a dearth of precise data in Iran, but the risk of health care-associated infections is known to be high (12). To the best of our knowledge, there is currently no systematic study on hand hygiene promotion and evaluation in health facilities in Iran. As a developing country, Iran has yet to collect information on how hand hygiene compliance affects the rates of health care-associated infections in its hospitals. Previous, unpublished observations from Iranian hospitals have indicated that hand hygiene compliance is poor among hospital staff, especially medical students.

\section{Objectives}

The objectives of this study were to provide an assessment of KAP regarding standard hand hygiene among medical residents in Imam Hossein hospital, Tehran, Iran, and to identify the determinants associated with them. Imam Hossein hospital has different student burdens and thus mainly relies on infection control techniques taught to the students as part of the clinical skills training. We measured the rates of compliance with hand hygiene after presenting an oral lecture and practical training on hand hygiene to the residents. The study employed the hand hygiene observation method of the world health organization (WHO).

\section{Patients and Methods}

This is a cross-sectional KAP study conducted between October and November 2013 among medical residents in Imam Hossein Hospital affiliated to Shahid Beheshti university of medical sciences, Tehran, Iran. Established in 1986, Imam Hossein hospital is a 570-bed educational and general medical/surgical referral hospital with general surgery, internal medicine, pediatrics, obstetrics and gynecology, neurology, infectious diseases, neurosurgery, ophthalmology, orthopedics, oncology, radiology, emergency, psychiatric, dialysis, and pathology wards as well as ICU, NICU, CCU, operating rooms, clinics, and a clinical laboratory.

The study explored KAP on the topic of hand hygiene practice among the medical residents. All the medical residents from the different wards were invited to participate in this study (270 in total). The participants were in different years of their residency period. Different age groups and both sexes were included. The purpose of the study was fully explained to the study participants, and informed consent was obtained. Confidentiality was maintained at all levels of the study by avoiding use of name and other identifiers. Participants' involvement in the study was on a voluntary basis: Participants who were unwilling to participate in the study and those who wished to discontinue their participation were allowed to do so without any restriction. The study protocol as well as the questionnaire was approved by the ethics committee of Shahid Beheshti university of medical sciences (ethics approval code:131/2013.03.02). Thereafter, an official letter was obtained from the administrative body of Imam Hossein hospital.

The volunteers $(\mathrm{n}=256)$ were divided into groups, each consisting of 15 members. The investigator visited the groups of volunteers in the hospital's hall and explained the nature of the study. The study was divided into 3 phases: evaluation, training, and direct observation.

\subsection{Evaluation Phase}

This phase assessed KAP on hand hygiene practices among the residents. The instrument used was the Hand Hygiene Knowledge Questionnaire for Health Care Workers, originally developed by the WHO (2009) (13). The questionnaire contains 25 items with both multiple choices and "Yes" or "No" questions in the English language but translated into the Persian language for proper understanding. The questionnaire administration took about 20 minutes to complete. The respondents were requested to complete the survey without discussing it with one another. The questions encompassed queries on washing hands or the use of alcohol gels, the use of gloves and masks, and the correct procedure for hand hygiene. There were no floor and ceiling effects.

Attitude was measured using 19 questions where the respondents were given the option to select on a 1- to 7-point scale between not effective and very effective or very low and very high. The instrument was the Questionnaire for perception survey for health care workers, originally developed by the WHO (2009).

The content validity of the questionnaire and checklist was determined through review by a panel of experts, who were asked to comment on whether the questionnaire adequately sampled hand hygiene knowledge; whether the questions and items in the questionnaire were accurate, clear, and easy to understand; whether the instructions were clear and complete; whether any of the questions or statements were likely to discour- 
age the participant from answering; and whether the response options were adequate for the questions and statements. The expert reviewers were also asked to give suggestions on possible improvements to the questionnaire and to comment on the relevance of the statements in the beliefs scale. Face validity was determined through 2 episodes of pilot testing the questionnaire on sixth-year medical students. The development and validation of the original instrument is described by the WHO (2009). As part of the validation in the Iranian setting, additional items from the original piloted version of the questionnaire were included in the scales for reliability testing. The reliability indices of the scales were assessed using Cronbach's alpha. The alpha value of the questionnaire was 0.77 in the pilot study and 0.75 in this study, which are considered $(14,15)$.

For the assessment of practice, a checklist was used to record practical activities performed by the residents. A specialized training session was conducted for the selected individuals who served as observers of hand hygiene compliance during the evaluation phase of the study. The observers were taught how to openly and objectively observe and monitor hand practices and to gather data on hand hygiene using the WHO hand hygiene methodology $(4,16)$. Time for the procedure, sequence, and steps of the hand hygiene procedure was recorded. Information on the residents' demographic variables and number of years on course was also collected.

\subsection{Training Phase}

The training phase included direct lectures to 256 residents concerning the WHO Guidelines on Hand Hygiene in Health Care and their implementation strategy and tools, including why, when, and how to perform hand hygiene in health care (15). The training programs were conducted to raise awareness among the residents in all specialties. The training sessions were conducted separately for the resident groups by the research team at the hospital's conference hall using PowerPoint presentation, and training handouts were given to each participant. Each training session was held once and lasted for $2 \frac{1}{2}-3$ hours and also involved groups comprising 15 residents per group. A total of 17 training sessions were held and finally a make-up session was held for the residents who for whatever reason were not involved.

\subsection{Direct Observation Phase}

During the third phase, hand hygiene compliance was evaluated by the trained observers using the direct observation technique described in the WHO Hand Hygiene Technical Reference Manual (17). To avoid a Hawthorne effect, where participant behavior is influenced by the awareness of the physical presence of the observer, the researcher was indistinguishable in the setting. The locations of the observations were prescheduled, and the observations were conducted daily, in prespecified 20-minute periods during the morning visit hours. The period of observation was formally announced to the head physician of the various wards. The observers were instructed to respect the patients' privacy and not interfere with the health care activities being carried out during the session. The observers were also instructed not to perform the observation in extreme situations (e.g. in high emergency medical treatment) since they may not reflect a "standard" care situation. All the observers stood or sat close to the point of care while observing: close enough to see but not interfere with patient care activities. Each observation form was checked immediately after the observation session and the end time, duration of the session, and signature were entered. The participants remained anonymous and unaware of the observers' presence.

Data cleanup and cross-checking was done before analysis. The data from the questionnaire were coded and entered into a computerized database and analyzed using statistical package for the social sciences (SPSS), version 17. Different KAP studies have drawn upon different analytic methods. In the present study, descriptive analysis was employed to characterize the demographic data and the responses given by the study groups. Bivariate analysis was used to assess unadjusted associations between the outcome variable (knowledge score) and the demographic characteristics. The $\chi^{2}$ test and the Fisher exact test were used to analyze the qualitative variables. Since the quantitative variables had no normal distribution, the Mann-Whitney test and the Kruskal-Wallis method were utilized. A P value $<0.05$ was considered statistically significant.

A scoring system was used where 1 point was awarded for each correct response to knowledge, positive attitudes, and good practices. Incorrect knowledge, negative attitudes, and poor practices were given 0 points. The cutoff values to determine good, moderate, and poor levels were taken from previously published studies with some modification to suit our purpose $(1,10)$. A score $>75 \%$ was considered good, $50 \%-74 \%$ moderate, and $<50 \%$ poor.

\section{Results}

\subsection{Demographic Data}

A total of 256 medical residents were recruited in the current study. The mean age ( \pm standard deviation [SD]) of the participants was $30.9 \pm 3.8$ years. Of the 256 respondents, 133 (52\%) were female and $123(48 \%)$ male. Of the entire study population, a minority $(16.4 \%, 42$ out of 256) claimed to have received formal training in hand hygiene.

The residents were receiving training in different specialties: internal medicine (28.5\%), emergency medicine (23.4\%), intensive critical care (3.1\%), surgery (11.7\%), obstetrics and gynecology (8.2\%), and pediatrics (4.3\%); and the 
remaining 20.7\% identified themselves as “other". Among the study population, 111 (43.4\%), 92 (35.9\%), 46 (18\%), and 7 (2.7\%) were in the first, second, third, and final years of the course, respectively.

\subsection{Knowledge about Hand Hygiene}

A total of 256 study residents were recruited using standard questions with a response rate of $100 \%$. Overall, the students' knowledge scores ranged from $8 / 25$ to $20 / 25$, and the mean score was $14.1 / 25$ ( \pm 2.6 ). In other words, the level of knowledge about hand hygiene was moderate (168 of $256,65.7 \%$ ) among the total study population. Only $4.3 \%$ of the participants (11 of 256 ) had good knowledge regarding hand hygiene, while $26.9 \%$ had moderate knowledge and the majority (67\%) had poor knowledge.

The mean scores of the residents' knowledge based on different categories are given in Table 1.

\subsection{Attitudes Toward Hand Hygiene}

The residents were surveyed on their attitude toward the effect of hand hygiene on health care-associated in- fections. Overall, the results were disappointing as the majority of the students had poor attitudes. Forty-nine percent ( $n=124$ ) of the residents responded to the questions on hand hygiene attitudes and only $20 \%(n=51)$ managed to identify the correct answer. The percentages of the respondents and the correct responses to the questions on hand hygiene attitudes based on different categories are demonstrated in Table 2.

\subsection{Practice of Hand Hygiene}

With respect to hand hygiene practices, only $3.1 \%(n=8)$ of the medical residents adhered to the 8 standard steps and only $12.1 \%(\mathrm{n}=31)$ washed their hands between 20 and 30 seconds. The residents' mean hand hygiene step was 4.5 , ranging from 1 to 8 . The mean hand hygiene time was 12 seconds, ranging from 10 to 16 . Additionally, only $2(0.8 \%)$ residents complied with the sequences of hand hygiene.

There was a significant difference in performance on hand hygiene between the residents on different years, while there was no significant difference in the category scores between the residents (Table 3 ).

\begin{tabular}{|c|c|c|c|c|c|}
\hline Parameter & Values ${ }^{b}$ & Mean $\pm S D$ & Median & IQR & P Value \\
\hline \multicolumn{6}{|l|}{ Age group, y } \\
\hline$\leq 30$ & $136(53.5)$ & $14.2 \pm 2.5$ & 14 & 12 to 16 & $0.737^{\mathrm{C}}$ \\
\hline$>31$ & $118(46.5)$ & $14 \pm 2.6$ & 14 & 12 to 16 & \\
\hline \multicolumn{6}{|l|}{ Gender } \\
\hline Female & $133(52)$ & $14.5 \pm 2.6$ & 15 & 12 to 16 & $0.023^{C}$ \\
\hline Male & $123(48)$ & $13.7 \pm 2.5$ & 14 & 12 to 16 & \\
\hline \multicolumn{6}{|l|}{ Specialty } \\
\hline Internal medicine & $73(28.5)$ & $14.4 \pm 2.7$ & 15 & 12 to 16 & $<0.001^{\mathrm{d}}$ \\
\hline Surgery & $30(11.7)$ & $13.6 \pm 2.6$ & 14 & 12 to 15 & \\
\hline ICU & $8(3.1)$ & $13.5 \pm 2.2$ & 13.5 & 11.5 to 15.5 & \\
\hline Other & $53(20.7)$ & $14.9 \pm 2.2$ & 15 & 14 to 17 & \\
\hline Obstetrics and gynecology & $21(8.2)$ & $15.6 \pm 2.5$ & 16 & 14 to 18 & \\
\hline Emergency medicine & $60(23.4)$ & $12.9 \pm 2$ & 13 & 12 to 14 & \\
\hline Pediatrics & $11(4.3)$ & $13.6 \pm 3.4$ & 14 & 10 to 16 & \\
\hline \multicolumn{6}{|l|}{ Year on the course } \\
\hline First & $111(43.4)$ & $14.1 \pm 2.5$ & 14 & 12 to 16 & $0.982^{\mathrm{d}}$ \\
\hline Second & $92(35.9)$ & $14.1 \pm 2.6$ & 14 & 12 to 16 & \\
\hline Third & $46(18)$ & $14.1 \pm 2.6$ & 14.5 & 12 to 16 & \\
\hline Fourth & $7(2.7)$ & $14.4 \pm 2.1$ & 15 & 13 to 16 & \\
\hline \multicolumn{6}{|c|}{ Taking training on hand hygiene } \\
\hline No & $214(83.6)$ & $13.9 \pm 2.5$ & 14 & 12 to 16 & $0.005^{c}$ \\
\hline Yes & $42(16.4)$ & $15.1 \pm 2.8$ & 16 & 13 to 17 & \\
\hline Total & $256(100)$ & $14.1 \pm 2.6$ & 14 & 12 to 16 & \\
\hline
\end{tabular}

a Abbreviation: IQR: Interquartile Range.

b Data are presented as No. (\%).

c Based on the Mann-Whitney test.

$\mathrm{d}$ Based on the Kruskal-Wallis test. 
Nabavi Met al.

Table 2. Percentages of the Respondents and the Correct Responses to the Questions on Hand Hygiene Attitudes Based on Different Categories $^{a}$

\begin{tabular}{|c|c|c|c|c|c|c|}
\hline Category & Total (\%) & Respondents (\%) & Correct Response (\%) & Median & IQR & PValue \\
\hline \multicolumn{7}{|l|}{ Age group, $y$} \\
\hline$\leq 30$ & $136(53.5)$ & $68(50)$ & $13(5.1)$ & 25 & 13 to 50 & $0.1^{b}$ \\
\hline$>31$ & $118(46.5)$ & $56(47)$ & $12(4.6)$ & 30 & 20 to 40 & \\
\hline \multicolumn{7}{|l|}{ Gender } \\
\hline Female & $133(52)$ & $64(48)$ & $13(5.1)$ & 30 & 20 to 50 & $0.1^{\mathrm{b}}$ \\
\hline Male & $123(48)$ & $60(49)$ & $12(4.6)$ & 30 & 15 to 40 & \\
\hline \multicolumn{7}{|l|}{ Specialty } \\
\hline Internal & $73(28.5)$ & $45(62)$ & $10(3.9)$ & 30 & 15 to 40 & $0.005^{C}$ \\
\hline Surgery & $30(11.7)$ & $19(63)$ & $5(1.9)$ & 25 & 20 to 45 & \\
\hline $\mathrm{ICU}$ & $8(3.1)$ & $2(25)$ & $1(0.4)$ & 50 & 20 to 80 & \\
\hline Other & $53(20.7)$ & $27(51)$ & $4(1.5)$ & 30 & 10 to 50 & \\
\hline Obstetrics and gynecology & $21(8.2)$ & $8(38)$ & $1(0.4)$ & 20 & 8 to 53 & \\
\hline Emergency & $60(23.4)$ & $21(36)$ & $3(1.2)$ & 25 & 20 to 40 & \\
\hline Pediatrics & $11(4.3)$ & $2(18)$ & $1(0.4)$ & 55 & 40 to 70 & \\
\hline \multicolumn{7}{|l|}{ Year on the course } \\
\hline First & $111(43.4)$ & $52(47)$ & $10(3.9)$ & 30 & 20 to 50 & $0.005^{C}$ \\
\hline Second & $92(35.9)$ & $44(48)$ & $8(3.1)$ & 25 & 13 to 40 & \\
\hline Third & $46(18)$ & $24(52)$ & $5(1.9)$ & 33 & 20 to 50 & \\
\hline Fourth & $7(2.7)$ & $4(57)$ & $2(0.8)$ & 28 & 20 to 33 & \\
\hline \multicolumn{7}{|c|}{ Taking training on hand hygiene } \\
\hline No & $214(83.5)$ & $99(46)$ & $10(3.9)$ & 30 & 15 to 40 & $0.04^{\mathrm{a}}$ \\
\hline Yes & $42(16.5)$ & $25(60)$ & $15(5.8)$ & 35 & 20 to 57 & \\
\hline Total & $256(100)$ & $124(49)$ & $25(9.7)$ & 30 & 20 to 40 & \\
\hline $\begin{array}{l}\text { a Abbreviation: IQR: Interquart } \\
\text { b Based on the Mann-Whitney } \\
\text { c Based on the Kruskal-Wallis te }\end{array}$ & & & & & & \\
\hline
\end{tabular}

Table 3. Mean Steps and Mean Times on Hand Hygiene for the Residents Based on Different Categories a,b

\begin{tabular}{|c|c|c|c|c|c|c|c|c|c|}
\hline \multirow[t]{2}{*}{ Parameter } & \multirow[t]{2}{*}{ Values $^{\mathrm{C}}$} & \multicolumn{4}{|c|}{ Steps } & \multicolumn{4}{|c|}{ Time } \\
\hline & & Mean \pm SD & Median & IQR & PValue & Mean \pm SD & Median & IQR & PValue \\
\hline \multicolumn{10}{|l|}{ Age group, y } \\
\hline$\leq 30$ & $136(53.5)$ & $4.5 \pm 1.5$ & 4 & 4 to 5 & $0.964^{\mathrm{d}}$ & $12 \pm 6$ & 10 & 10 to 15 & $0.121^{d}$ \\
\hline$>31$ & $118(46.5)$ & $4.5 \pm 1.3$ & 4 & 4 to 5 & & $13 \pm 5$ & 10 & 10 to 15 & \\
\hline \multicolumn{10}{|l|}{ Gender } \\
\hline Female & $133(52)$ & $4.5 \pm 1.4$ & 4 & 4 to 5 & $0.757^{\mathrm{d}}$ & $12 \pm 6$ & 10 & 10 to 15 & $0.907^{\mathrm{d}}$ \\
\hline Male & $123(48)$ & $4.5 \pm 1.4$ & 4 & 4 to 5 & & $12 \pm 5$ & 10 & 10 to 15 & \\
\hline \multicolumn{10}{|l|}{ Specialty } \\
\hline Internal & $73(28.5)$ & $4.3 \pm 1.2$ & 4 & 4 to 5 & $0.007^{\mathrm{e}}$ & $12 \pm 5$ & 10 & 10 to 15 & $0.039^{\mathrm{e}}$ \\
\hline Surgery & $30(11.7)$ & $5.4 \pm 1.6$ & 6 & 4 to 7 & & $13 \pm 4$ & 15 & 10 to 15 & \\
\hline ICU & $8(3.1)$ & $3.9 \pm 1.6$ & 4 & 3 to 5 & & $17 \pm 10$ & 18 & 8 to 25 & \\
\hline Other & $53(20.7)$ & $4.3 \pm 1.5$ & 4 & 3 to 5 & & $10 \pm 5$ & 10 & 5 to 15 & \\
\hline Obstetrics and gynecology & $21(8.2)$ & $4.9 \pm 1.9$ & 5 & 4 to 6 & & $12 \pm 8$ & 10 & 5 to 10 & \\
\hline Emergency & $60(23.4)$ & $4.4 \pm 1$ & 4 & 4 to 5 & & $13 \pm 4$ & 13 & 10 to 15 & \\
\hline Pediatrics & $11(4.3)$ & $5.1 \pm 1.4$ & 5 & 4 to 6 & & $14 \pm 10$ & 10 & 5 to 20 & \\
\hline \multicolumn{10}{|l|}{ Year on the course } \\
\hline First & $111(43.4)$ & $4.4 \pm 1.5$ & 4 & 4 to 5 & $0.081^{\mathrm{e}}$ & $12 \pm 6$ & 10 & 5 to 15 & $0.034^{\mathrm{e}}$ \\
\hline Second & $92(35.9)$ & $4.4 \pm 1.5$ & 4 & 3 to 5 & & $12 \pm 5$ & 10 & 10 to 15 & \\
\hline Third & $46(18)$ & $4.8 \pm 1$ & 5 & 4 to 6 & & $13 \pm 5$ & 15 & 10 to 15 & \\
\hline Fourth & $7(2.7)$ & $4.9 \pm 1.1$ & 5 & 4 to 5 & & $14 \pm 3$ & 15 & 10 to 15 & \\
\hline \multicolumn{10}{|c|}{ Taking training on hand hygiene } \\
\hline No & $214(83.6)$ & $4.4 \pm 1.3$ & 4 & 4 to 5 & $0.085^{\mathrm{d}}$ & $12 \pm 6$ & 10 & 10 to 15 & $0.274^{d}$ \\
\hline Yes & $42(16.4)$ & $4.9 \pm 1.7$ & 5 & 4 to 6 & & $13 \pm 6$ & 15 & 10 to 15 & \\
\hline Total & $256(100)$ & $4.5 \pm 1.4$ & 4 & 4 to 5 & & $12 \pm 6$ & 10 & 10 to 15 & \\
\hline
\end{tabular}

a Standard steps $=8$ and Standard time $=20-30$ seconds.

b Abbreviation: IQR: Interquartile Range.

c Data are presented as No.(\%).

d Based on the Mann-Whitney test.

e Based on the Kruskal-Wallis test. 


\subsection{Direct Observation}

The observation was carried out among the same residents $(\mathrm{n}=256$ ) during the morning visit hours in the ward. It was observed that none of the residents performed hand washing with available means (water and hand-washing liquid) in the morning visit hours.

\section{Discussion}

Although hand hygiene is a very simple procedure and has long been deemed one of the most important infection control measures, the compliance rates by health care workers are generally reported to be low $(1,9)$.

The results of the current study revealed that all the Iranian residents had poor hand hygiene compliance. This finding is lower than that reported in other studies done via a similar method in Asian countries like Kuwait and India $(16,18)$. This may be due to a lack of hand hygiene resources in our hospitals, specifically Imam Hossein hospital, and there may be insufficient knowledge about hand hygiene. An alternative explanation is that although guidelines are simple and easy to learn, translating them into practice in a working environment is a challenge. And this is while poor hand hygiene compliance on the part of health care workers and its complication of health care-associated infections have impact on patients, visitors, and health care workers (6).

In the present study, the medical residents had moderate knowledge about hand hygiene, which was a positive finding. In a similar survey by Colosi et al. Italian health care students' knowledge mean score was better than that of Iranian residents (19).

Knowledge about hand hygiene was found to be significantly better among the obstetrics and gynecology medical residents when compared to the others. Their better score may be due to a good preclinical skills training program. The low level of knowledge of hand hygiene among the emergency medical residents may be attributed to their poor educational background and non-provision of this information by their immediate superior. The existing literature contains no similar study for comparison; nonetheless, previous observational studies have found that nurses tend to have better hand hygiene practices than doctors $(20,21)$.

The female medical residents' mean knowledge score was higher than that of their male counterparts. There is considerable evidence to confirm our finding (22). Training in hand hygiene was found to be significantly associated with the residents' knowledge of hand hygiene. Those who were trained had more compliance than those who were not trained. This finding also chimes in with some other investigations conducted in the United Kingdom and China showing that training had a positive relationship with hand hygiene compliance in all medical staff $(9,23)$. This may be due to the fact that training builds the capacity of medical residents, which has a significant association with hand hygiene compliance.
Indeed, training can be crucial in terms of hand hygiene compliance; and by extension, post-training follow-up may contribute to better hand hygiene.

In the current survey, the participants' level of attitude toward the various aspects of health care-associated in fections was low inasmuch as only 25 (20.16\%) residents were aware about some infections that a health care worker can acquire from a patient. The failure of over $50 \%$ of the residents to answer the question is worrisome. Also, there were wide areas where the level of attitude was low, particularly regarding infections that a health care worker can transmit to a patient $(8,10,20)$. Consequently, this specific population needs to receive far better training vis-à-vis hand hygiene if the rates of health care-associated infections are to be lowered. In our study, the surgery and internal medicine residents showed better attitudes toward hand hygiene than the residents of other specialties. This finding demonstrated that the Iranian final-year medical residents and also the medical residents who were trained had better attitudes toward hand hygiene.

In the current study, although the Iranian medical residents had moderate knowledge about hand washing concepts, they failed to apply proper hand hygiene techniques. An unexpected finding was that none of the residents adhered to the standards of time, sequence, and steps of hand hygiene simultaneously. It has been suggested that the optimal duration of hand washing is between a minimum of 20 seconds and a maximum of 30 seconds. However, only a few medical residents (12.1\%) observed the optimal time in the hand hygiene procedure. The majority of the residents cited time constraint as the salient reason for their failure to comply with the hand hygiene procedure (data not shown). Similar findings were reported by some previous studies among health care workers in that they associated their high workload with their non-adherence to hand hygiene $(1,24)$.

Our results showed that only 8 residents washed their hands in 8 steps, the standard steps required for effective hand hygiene as documented in the WHO guidelines on hand hygiene in health care. Furthermore, $99.2 \%$ of them could not correctly identify the sequence of effective hand washing. This finding is similar to the results of a previous investigation reporting that $91.4 \%$ of its study population could not correctly identify the sequence of effective hand washing (25). Our findings also revealed that despite having a satisfactory level of knowledge and awareness about hand washing, the medical residents in Imam Hossein Hospital had poor hand hygiene practice, which may be due to possible inadequate hygiene supervision in the hospital.

In the present study, we held a training session to improve the residents' KAP concerning hand hygiene in order to ensure that whatever was learned would be put into practice. Our findings are in agreement with those reported by some previous studies $(26,27)$ insofar as training and knowledge, albeit fundamental, are not suf- 
ficient to foster a behavioral change with respect to hand hygiene. As regards the practice of hand washing before performing medical procedures in the morning visit hours, none of the residents washed their hands. This observation is concordant with the findings of some other studies $(4,23,24)$ showing that improvement in hand hygiene compliance remained low among doctors and other health care workers. It is important to carry out training programs on hand hygiene regularly for health care workers as it has been associated with increased compliance with hand hygiene practices and reduction of infection (21, 23). Hand hygiene training sessions should be conducted more frequently for medical students with continuous monitoring and performance feedback to encourage them to observe correct hand hygiene practices. As doctors are the key players in the health care team, it is vitally important to provide them with optimal training to raise their knowledge regarding the preventive measures against infectious diseases. A study on hand hygiene among final-year medical students suggested that poor compliance might have its roots in a failure to learn this behavior at medical school (28). Better education via a multifaceted approach in hospital policies is required if KAP regarding infection control practices is to be bettered. However, no clear evidence exists as to what constitutes the best way to teach or assess the effectiveness of infection control training, despite recommendations that it should be included in the formal medical and health sciences curriculum (11).

The level of hand hygiene compliance among the Iranian residents in the present study is alarmingly low. Be that as it may, a review of literature clearly demonstrates a similarly dismal picture painted by other studies. The situation in the health care centers of developing countries is even more unacceptable $(16,18,20)$. All these findings highlight the need for the current training programs to target hand hygiene practices among medical students. Fortunately, compliance with hand hygiene among health care workers can be enhanced significantly through regular hand hygiene campaigns using posters and encouraging peers to remind colleagues of hand hygiene (23).

There are many issues concerning all aspects of hand hygiene which remain unresolved. While hand hygiene practices are simple, compliance with hand hygiene falls in the domain of human behavior; and altering human behavior is complex and constitutes an enormous challenge. This study was conducted in a large number of wards with various designated specialties in a referral hospital in the capital of Iran, Tehran. Our findings may, therefore, be generalized to all medical degree graduates who start internships in different designated hospitals across the country. As the structures of the curricula and system of most Iranian medical universities are similar, our findings should serve as a basis for future research in medical education and health care in Iran. The absence of such information hampers the development of effec- tive policies on hand hygiene both at national and local levels. The findings of this study could serve as useful evidence for scholars interested in this field.

The Iranian medical residents recruited in the present study had moderate knowledge about hand hygiene. However, their levels of attitudes and practices were unsatisfactory. This result demonstrates the low rate of hand hygiene compliance among Iranian medical residents due to substantial deficiencies in their learning resources. Multifaceted and dedicated efforts must be undertaken to rectify this attitude and behavior from the onset. Indeed, we would highly recommend that medical schools seek to modify and enrich their curriculum in order to improve hand hygiene practices among their students. An improved understanding of infection control and hand hygiene among medical students could play a major role in curbing disease transmission when the current medical students graduate and join the health care work force in the future. A repeat of this study covering a longer period of time is needed to confirm the observed results.

\section{Acknowledgements}

The authors are grateful to the medical residents and staff in Imam Hossein hospital who facilitated the survey, and resident volunteers for taking part in the study. This study was supported by Shahid Beheshti university of medical sciences (grant \# 1071/1391). The funders had no role in the study design, data collection and analysis, decision to publish, or preparation of the manuscript.

\section{Authors' Contributions}

Study concept and design: Mahmoud Nabavi and Mohammad Moeinian. Acquisition of the data: Mohammad Moeinian and Latif Gachkar. Analysis and interpretation of the data: Mostafa Alavi-Moghadam and Mohammad Moeinian. Drafting of the manuscript: Mohammad Moeinian. Critical revision of the manuscript for important intellectual content: Mahmoud Nabavi, Latif Gachkar, and Mostafa Alavi-Moghadam. Statistical analysis: Latif Gachkar and Mohammad Moeinian. Administrative, technical, and material support: Mahmoud Nabavi and Mohammad Moeinian. Study supervision: Mahmoud Nabavi.

\section{Funding/Support}

The current cross-sectional knowledge, attitudes, and practices study was financially supported by Shahid Beheshti University of Medical Sciences.

\section{References}

1. Allegranzi B, Bagheri Nejad S, Combescure C, Graafmans W, Attar $\mathrm{H}$, Donaldson L, et al. Burden of endemic health-care-associated infection in developing countries: systematic review and metaanalysis. Lancet. 2011;377(9761):228-41.

2. Mathai E, Allegranzi B, Kilpatrick C, Pittet D. Prevention and control of health care-associated infections through improved hand hygiene. Indian J Med Microbiol. 2010;28(2):100-6. 
3. Pittet D, Allegranzi B, Boyce J, World Health Organization World Alliance for Patient Safety First Global Patient Safety Challenge Core Group of E. The World Health Organization Guidelines on Hand Hygiene in Health Care and their consensus recommendations. Infect Control Hosp Epidemiol. 2009;30(7):611-22.

4. Haas JP, Larson EL. Measurement of compliance with hand hygiene. J Hosp Infect. 2007;66(1):6-14.

5. Day M. Doctors attack Gordon Brown's plans to regulate medical profession. BMJ. 2007;335(7611):113.

6. Erasmus V, Brouwer W, van Beeck EF, Oenema A, Daha TJ, Richardus $\mathrm{JH}$, et al. A qualitative exploration of reasons for poor hand hygiene among hospital workers: lack of positive role models and of convincing evidence that hand hygiene prevents crossinfection. Infect Control Hosp Epidemiol. 2009;30(5):415-9.

7. Jang JH, Wu S, Kirzner D, Moore C, Youssef G, Tong A, et al. Focus group study of hand hygiene practice among healthcare workers in a teaching hospital in Toronto, Canada. Infect Control Hosp Epidemiol.2010;31(2):144-50.

8. Patarakul K, Tan-Khum A, Kanha S, Padungpean D, Jaichaiyapum OO. Cross-sectional survey of hand-hygiene compliance and attitudes of health care workers and visitors in the intensive care units at King Chulalongkorn Memorial Hospital. J Med Assoc Thai. 2005;88 Suppl 4:S287-93.

9. Yuan CT, Dembry LM, Higa B, Fu M, Wang H, Bradley EH. Perceptions of hand hygiene practices in China. $J$ Hosp Infect. 2009;71(2):157-62.

10. Ariyarathne M, Gunasekara T, Weerasekara MM, Kottahachchi J, Kudavidanage BP, Fernando SSN. Knowledge, attitudes and practices of hand hygiene among final year medical and nursing students at the University of Sri Jayewardenepura. Sri Lankan J of Infectious Dis. 2013;3(1):15-25.

11. Chan MF, Ho A, Day MC. Investigating the knowledge, attitudes and practice patterns of operating room staff towards standard and transmission-based precautions: results of a cluster analysis. J Clin Nurs. 2008;17(8):1051-62.

12. Naderi HR, Sheybani F, Mostafavi I, Khosravi N. Compliance with hand hygiene and glove change in a general hospital, Mashhad, Iran: an observational study. Am J Infect Control.2012;40(6):e221-3.

13. WHO 2013 Available from: http://www.who.int/gpsc/5may/tools evaluation_feedback/en/http://www.who.int/gpsc/5may/tools.

14. Abdulraheem IS, Amodu MO, Saka MJ, Bolarinwa OA, Uthman MMB. Knowledge, Awareness and Compliance with Standard Precautions among Health Workers in North Eastearn Nigeria. J Community Med Health Edu. 2012;2(131):2.

15. Sax H, Allegranzi B, Chraiti MN, Boyce J, Larson E, Pittet D. The
World Health Organization hand hygiene observation method. Am J Infect Control. 2009;37(10):827-34

16. Al-Wazzan B, Salmeen Y,Al-Amiri E, Abul A, Bouhaimed M,Al-Taia A. Hand hygiene practices among nursing staff in public secondary care hospitals in Kuwait: self-report and direct observation. Med Princ Pract. 2011;20(4):32 6-31.

17. Larson EL, Aiello AE, Cimiotti JP. Assessing nurses' hand hygiene practices by direct observation or self-report. J Nurs Meas. 2004;12(1):77-85.

18. Sharma S, Sharma S, Puri S, Whig J. Hand hygiene compliance in the intensive care units of a tertiary care hospital. Indian J Community Med. 2011;36(3):217-21.

19. Colosi A, Ergasti G, Murzilli G, Paolini V, Semeraro V, Trapani MM et al. Healthcare students and their knowledge of healthcareassociated infections. Ann Ig. 2011;23(3):203-8.

20. Abd Elaziz KM, Bakr IM. Assessment of knowledge, attitude and practice of hand washing among health care workers in Ain Shams University hospitals in Cairo. J Prev Med Hyg. 2009;50(1):19-25

21. Salemi C, Canola MT, Eck EK. Hand washing and physicians: how to get them together. Infect Control Hosp Epidemiol. 2002;23(1):32-5.

22. Moret L, Tequi B, Lombrail P. Should self-assessment methods be used to measure compliance with handwashing recommendations? A study carried out in a French university hospital. Am J Infect Control. 2004;32(7):384-90.

23. Randle J, Clarke M, Storr J. Hand hygiene compliance in healthcare workers. J Hosp Infect. 2006;64(3):205-9.

24. Pittet D, Hugonnet S, Harbarth S, Mourouga P, Sauvan V, Touveneau $S$, et al. Effectiveness of a hospital-wide programme to improve compliance with hand hygiene. Infection Control Programme. Lancet. 2000;356(9238):1307-12.

25. Aznal SS, Yun LW, Joo CL, Liang EW, Suleiman MF, Fook TC. The lack of practice in effective hand washing against the actual high level of knowledge and awareness among medical students during clinical practice.

26. Akyol A, Ulusoy H, Ozen I. Handwashing: a simple, economical and effective method for preventing nosocomial infections in intensive care units. J Hosp Infect. 2006;62(4):395-405.

27. Tipple AFV, Mendonça KM, de Melo MC, Silva AC, Pereira MS, dos Santos SL. Higienização das mãos: o ensino e a prática entre graduandos na área da saúde-DOI:10.4025/actascihealthsci.1079. Acto Scientiarum. Health Sci. 2008;29(2):107-14.

28. Lankford MG, Zembower TR, Trick WE, Hacek DM, Noskin GA, Peterson LR. Influence of role models and hospital design on hand hygiene of healthcare workers. Emerg Infect Dis. 2003;9(2):217-23. 